\title{
Assessment of regional capabilities for agricultural coexistence with genetically modified maize
}

\author{
Angelika Wurbs*', Claudia Bethwell ${ }^{*}$ and Ulrich Stachow
}

\begin{abstract}
Background: The coexistence of agricultural production with and without the use of genetically modified (GV) crops is supposed to be made possible in Germany by regulations, which include minimal distances of GV-fields to potentially susceptible crop fields and habitats. To explore the impact of these regulations on region specific coexistence potentials, we broadened the applicability of an existing method for the simulation of the spatial distribution of arable fields cropped with conventional, organic and GV-maize. We used simulations which combine a variety of minimum distances of GV-maize fields to assess regional specific options and limitations for coexistence.

Results: An existing method was extended to be applicable for different spatial scales, from the large (e.g. Federal State) to small (e.g. municipality). Input data consisted of cropping statistics, geometry of arable fields and protected areas. Scenarios of cropping situations included various minimal distances between GV-maize fields and protected areas and various proportions of maize within the areas. The results of the simulations represent possible distribution patterns of non-GV and GV-maize fields as well as the size of the remaining area in which additional GV-maize can be grown without violating the minimal distance rules. As suspected, increasing proportions of GVmaize and increasing minimal distances lower the areas suitable for additional GV-maize. However, the relation between the area of GV-maize grown and those suitable for GV-maize cultivation varied between the scenarios. Moreover, the variability between the municipalities was even more evident, due to varying landscape structure (proportion of maize, the ratio total arable land to maize, proportion of protected areas). Areas with high proportions of GV-maize, of protected areas and of maize could be problematical for coexistence. We discuss these parameters with regard to other coexistence studies.

Conclusions: Our method is suitable to simulate the spatial distribution of fields cultivated with GV-crops and nonGV-crops on various scales. Simulations on the scale of a Federal State reveals those areas, in which coexistence could be problematical. Simulations on a county scale, however, allow more insight into options and restrictions for coexistence in relation to landscape structural characteristics, which also can be transferred to larger scales. On the scale of municipalities simulations can help to analyse the limits of coexistence in areas of high conflict potential, moreover this level is more realistic with regard to practical agricultural decisions on the farm level.
\end{abstract}

\footnotetext{
* Correspondence: awurbs@zalf.de; Claudia.Bethwell@zalf.de

Leibniz-Zentrum für Agrarlandschaftsforschung (ZALF) e.V., Institut für

Landnutzungssysteme, Eberswalder Straße 84, Müncheberg, D-15374,

Germany
} 


\section{Zusammenfassung}

Hintergrund: Die Koexistenz verschiedener landwirtschaftlicher Produktionsformen - mit und ohne Anbau von gentechnisch veränderten Pflanzen (GVO) - soll durch gesetzlich fixierte Regeln ermöglicht werden, die unter anderem die Mindestabstände von GVO-Anbauflächen zu potenziell empfindlichen anderen Ackerflächen und Biotopen festlegen. Hier wurde eine Methode weiterentwickelt zur Simulation der räumlichen Verteilung der Anbauflächen von konventionellem, ökologischem und GV-Mais um regionsspezifische Koexistenzpotenziale von gentechnisch verändertem Mais, sowie potenzielle Konfliktgebiete zu identifizieren.

Ergebnisse: Eine für Brandenburg entwickelte GIS-Simulationsmethode wurde durch die Verwendung flächendeckend vorhandener Daten so erweitert, dass sie bundesweit übertragbar ist und auf unterschiedlichen räumlichen Skalenebenen angewandt werden kann, von großräumig (Bundesland) bis lokal (Gemeinde). Als Eingangsdaten wurden Anbaustatistiken sowie Geometrien der Ackerflächen und von FFH- und Naturschutzgebieten verwendet. In den Szenarien wurden Abstandsregelungen zwischen Maisanbauflächen und zu Schutzgebieten und der GV-Maisanteil variiert. Die Ergebnisse der Simulation sind mögliche räumliche Verteilungen von Nicht-GV-Mais und GV-Mais sowie die potenziell für den Anbau von GV-Mais zur Verfügung stehende Fläche. Mit zunehmendem GV-Maisanteil und Mindestabständen zu Schutzgebieten wird die für den GV-Mais zur Verfügung stehende Fläche stärker ausgeschöpft. Der Anteil des GV-Mais an der potenziell für den Anbau von GVMais zur Verfügung stehenden Fläche variierte zwischen den Szenarien, und noch stärker jedoch regional zwischen den Landkreisen, verursacht durch deren verschiedene agrar- und landschaftsstrukturelle Ausstattung (Maisanbauanteil, Verhältnis Ackerlandsanteil/Maisanbauanteil, Schutzgebietsanteil). Ein räumliches Konfliktpotenzial bei der Umsetzung der Koexistenz ist in Gebieten hohen Nutzungsdrucks zu erwarten, d.h. in denen sowohl der Maisanbauanteil an der Ackerfläche und der Anbauanteil von GV-Mais, als auch der Schutzgebietsanteil hoch sind. Diese Faktoren werden diskutiert in Bezug zu Ergebnissen weiterer Koexistenzstudien.

Schlussfolgerungen: Die vorgestellte Methode ist geeignet, die räumliche Verteilung des Anbaus von Nicht-GVMais und GV-Mais auf unterschiedlichen Skalenebenen zu simulieren: Die Ebene eines Bundeslandes liefert Hinweise auf Gebiete, in denen die Koexistenz problematisch sein könnte und kann als Grundlage weiterer Berechnungen, wie zum Beispiel der Modellierung von Genflüssen auf Landesebene dienen. Die Simulation auf der Ebene eines Landkreises oder einer Gemeinde ermöglicht genauere Aussagen über die Möglichkeiten und Grenzen der Koexistenz. Auf der Ebene der Landkreise können z.B. unterschiedliche agrar- und landschaftsstrukturelle Situationen untersucht und für eine nachfolgende Regionalisierung angewandt werden. Die Ebene der Gemeinden erlaubt die Analyse der Grenzen der Koexistenz für Gebiete mit höherem räumlichen Konfliktpotenzial. Simulationen auf lokaler Ebene erscheinen darüber hinaus näher an den Entscheidungsmöglichkeiten der landwirtschaftlichen Praxis.

Keywords: GM-crops, Genetically modified crops, Bt-maize, coexistence, maize, simulation model, GIS

\section{Hintergrund}

Nach der EU-Richtlinie 2001/18/EC ${ }^{1}$ können die Mitgliedsstaaten der EU Maßnahmen zur Verhinderung des unbeabsichtigten Vorhandenseins von GVO ergreifen und damit die Koexistenz von genetisch veränderten und konventionellen und ökologischen Kulturen ermöglichen, welche in Deutschland durch das GenTG $^{2}$ und die GenTPflEV $^{3}$ umgesetzt werden. Demnach gilt für den Maisanbau in Deutschland, dass eine Koexistenz zwischen konventionell, ökologisch und mit Mitteln der Gentechnik erzeugtem Mais (GV-Mais) gewährleistet werden muss. Für den Anbau und für die Weiterverarbeitung soll nach dem GenTG ${ }^{2}$ eine Entscheidungsfreiheit der Landwirte und Verbraucher gewährleistet werden. Für den Maisanbau in einer Landschaft soll damit sowohl das Nebeneinander der Produktionsverfahren unter den gegebenen räumlichen Bedingungen ermöglicht als auch die Einkreuzung von GV-Mais in Nicht-GV-Mais vermieden werden.
In dieser Arbeit wird eine Methode dargestellt, und deren Ergebnisse mit anderen Arbeiten verglichen [1-2], mit der die räumliche Verteilung von Maisanbauflächen, auch von GV-Sorten, in einer Region simuliert werden kann. Die Ergebnisse können zunächst Aussagen darüber liefern, in welchem Umfang das räumliche Nebeneinander von Nicht-GV-Mais und GV-Mais in einer Region möglich und in welchen Gebieten Koexistenz problematisch ist. Koexistenz wird in Deutschland geregelt durch Mindestabstände zwischen GV-Maisanbauflächen und Nicht-GV-Maisflächen. So muss nach der GenTPflEV ${ }^{3}$ ein Mindestabstand von $150 \mathrm{~m}$ zu Nicht-GV-Maisflächen eingehalten werden, bzw. von $300 \mathrm{~m}$, wenn es sich um ökologischen Anbau handelt.

Die Festlegung von Mindestabständen wird auch in anderen europäischen Ländern als eine Maßnahme zur Gewährleistung der Koexistenz eingesetzt [3-4].

Neben den Mindestabständen zu Nicht-GV-Maisflächen ist als weitere Landschaftskomponente, die die Verteilung 
von GV-Mais Anbauflächen mitbestimmen kann, die Schutzgebiete (Naturschutzgebiete und FFH-Gebiete) zu beachten. Sie unterliegen in einigen Bundesländern besonderen Bestimmungen hinsichtlich des Anbaus von GV-Mais. In Brandenburg beispielsweise sollte der Abstand zu FFH-Gebieten mindestens $800 \mathrm{~m}$ betragen, sonst ist im Einzelfall eine naturschutzfachliche Verträglichkeitsprüfung vorgesehen und für NSG wird bei Unterschreitung des $800 \mathrm{~m}$ Abstandes eine Untersagungsanordnung geprüft ${ }^{4}$. Dies wird damit begründet, dass auch der Pollen von GVMais (im speziellen Fall Bt-Mais MON 810) das Eiweiß $\mathrm{Cry} 1 \mathrm{Ab}$ enthält, welches toxisch auf den Maisschädling Ostrinia nubilalis (Maiszünsler, ein Schmetterling) wirkt. Da Maispollen u.U. durch den Wind über mehrere hundert Meter verfrachtet werden kann, und z.Zt. nicht ausgeschlossen werden kann, dass das Cry1Ab auch toxisch auf andere Schmetterlingsarten wirkt, besteht hier möglicherweise ein Risiko gegenüber Naturschutzzielen. Im BayNatSchG ${ }^{5}$ wurde ein Prüfradius von $1000 \mathrm{~m}$ um Natura 2000 - Gebiete festgelegt. Ein beabsichtigter Anbau kann nur dann innerhalb dieses Radius stattfinden, wenn eine mögliche Gefährdung von Schutzzielen im Rahmen einer entsprechenden Prüfung ausgeschlossen werden konnte. Anbaubeschränkungen von GV-Mais durch Mindestabstände $\mathrm{zu}$ anderen Flächennutzungen bilden Restriktionen, die sich auf die räumliche Verteilung potenzieller Anbauflächen auswirken.

Die Simulationsergebnisse sind darüber hinaus eine wichtige Grundlage für die Modellierung von Genflüssen zwischen Maisanbauflächen. Diese kann Problemgebiete ausfindig machen, in denen die räumliche Verteilung der Maisanbauflächen eine Überschreitung von Grenzwerten hinsichtlich der Einkreuzung von GV-Material in konventionelle (d.h. Nicht-GVO) Sorten wahrscheinlich macht. Es sind zwar die o.g. Mindestabstände zwischen GV-Maisanbauflächen und Nicht-GV-Maisflächen in der GenTPflEV vorgeschrieben, mit denen Überschreitungen verhindert werden sollen, doch auch bei Beachtung dieser Mindestabstände können GV-Mais-Anbauer zu Schadenersatzzahlungen verpflichtet werden, wenn in benachbarten Nicht-GV-Maisflächen Grenzwerte überschritten werden. Durch Modellierungen auf Landschaftsebene können Gebiete identifiziert werden, in denen es trotz Einhaltung der Mindestabstände $\mathrm{zu}$ Grenzwert überschreitenden Einkreuzungen beim Anbau von GVMais kommen könnte [5].

Es ist zu erwarten, dass sich sowohl die Flächenanteile als auch die Geometrien (Größe, Lage) von Maisanbauflächen und von Schutzgebieten in einer Landschaft auf die potenziellen Koexistenz-Problemgebiete auswirken. Hinweise hierzu liefern z.B. Ergebnisse der Studie über Brandenburg von Höltl und Wurbs (2008) [1].

Die Simulation der räumlichen Verteilung von Maisanbauflächen und die damit verbundene Darstellung potenzieller Konfliktgebiete beim Anbau von GV-Mais kann auf verschiedenen räumlichen Skalenebenen durchgeführt werden, mit denen jeweils unterschiedliche Fragen beantwortet werden. Auf Basis der räumlichen Verteilung von Maisanbauflächen mit unterschiedlichen Wirtschaftsweisen (konventionell, ökologisch, GV) können z.B. für ganze Bundesländer die Anbaupotenziale für GV-Mais abgeschätzt und verglichen werden, sowie großräumig der Genfluss zwischen den Maisanbauflächen modelliert werden. Auf der Ebene eines Landkreises können regionale Anbaupotenziale abgeschätzt werden, die Hinweise zu möglichen räumlichen Konflikten geben und auch regionale Modellierungen des Genflusses erlauben. Auf der Gemeindeebene ist die Simulation darüber hinaus geeignet, lokale Kapazitätsgrenzen des Anbaus von GV-Mais zu bestimmen und zur Lösung von Koexistenzproblemen beizutragen. Anhand exemplarischer Simulationen auf diesen drei Ebenen werden mögliche Interpretationen der Simulationsergebnisse, ihre Grenzen und damit die Auswahl der geeigneten Simulationsebene zur Beantwortung unterschiedlicher Fragestellungen und praktische Anwendungsfälle diskutiert.

\section{Methode}

\section{Allgemeine Vorgehensweise und Eingangsdaten}

Die Methode basiert auf der von Höltl und Wurbs (2008) [1] für Brandenburg entwickelten Vorgehensweise zur Simulation des Anbaus von GV-Mais unter verschiedenen Koexistenzregelungen und wird dementsprechend umgesetzt mit einem GIS-Algorithmus, der die Verteilung der Maisanbauflächen simuliert. Datenvorbereitung und Simulation erfolgten mit ArcGIS 9.2 und ArcINFO von ESRI. Die Methode wurde hier weiterentwickelt, so dass sie nun bundesweit und auf verschiedenen räumlichen Ebenen angewendet werden kann. Die Weiterentwicklung betraf die Auswahl der Eingangsdaten, die Anpassung und Vorbereitung der Eingangsdaten und einzelne Schritte der eigentlichen Simulation.

Alle Eingangsdaten wurden so ausgewählt, dass sie für eine bundesweite Simulation übertragbar sind und wurden hier auf die Fallstudie Bundesland Bayern angewandt. Für die Simulation der Maisanbauflächen im Bundesland Bayern wurden die in Tabelle 1 gelisteten Eingangsdaten verwendet [6-11], wie statistische Daten zum Maisanbau, Geodaten über Ackerflächen und Schutzgebiete, sowie Verwaltungsgrenzen.

Weiterhin war eine Anpassung der Eingangsdaten und die Vorbereitung dieser Daten vor der eigentlichen Simulation notwendig. Das betraf zunächst die Daten zum Maisanbau in Deutschland. Sie sind in einigen Bundesländern nur auf der Ebene des Landkreises und in der überwiegenden Zahl der Bundsländer auf der Ebene der Gemeinde verfügbar. Die von [1] entwickelte Simulation wurde am Beispiel des Bundeslandes 
Tabelle 1 Eingangsdaten zur Simulation der Maisanbauflächen für das Bundesland Bayern

\begin{tabular}{|c|c|c|c|}
\hline Parameter & Eingangsdaten & Format & Datenquelle \\
\hline \multirow[t]{3}{*}{ Maisanbau } & Silomais & Agrarstatistik,ASE 2007 & $\begin{array}{l}\text { Statistische Ämter des Bundes und der } \\
\text { Länder (2009) [7]: Tabelle 115-02 }\end{array}$ \\
\hline & Körnermais & Agrarstatistik,ASE 2007 & $\begin{array}{l}\text { Bayrisches Landesamt für Statistik und } \\
\text { Datenverarbeitung } \\
\text { (2009): [6] Tabelle 41121-204r }\end{array}$ \\
\hline & ökologisch angebauter Mais & Agrarstatistik,ASE 2007 & $\begin{array}{l}\text { Statistische Ämter des Bundes und der } \\
\text { Länder (2009) [8]: } \\
\text { Tabelle 115-34-4 }\end{array}$ \\
\hline Verwaltungsgrenzen & $\begin{array}{l}\text { Bundesland, Landkreis, } \\
\text { Gemeinde }\end{array}$ & Geodaten & $\begin{array}{l}\text { Vermessungsverwaltungen der Länder } \\
\text { und BKG (2007) [9] }\end{array}$ \\
\hline $\begin{array}{l}\text { Geometrie der } \\
\text { Ackerflächen }\end{array}$ & $\begin{array}{l}\text { Ackerfläche (ATKIS } \\
\text { Objektart 4101) }\end{array}$ & Geodaten & $\begin{array}{l}\text { Vermessungsverwaltungen der Länder } \\
\text { und BKG (2008) [10] }\end{array}$ \\
\hline Schutzgebiete & NSG,FFH-Gebiete & Geodaten & Bayerisches Landesamt für Umwelt (2009) [11] \\
\hline
\end{tabular}

Brandenburg entwickelt, in dem die Maisanbaudaten auf der Ebene des Landkreises vorliegen. Diese Landkreise wurden von [1] differenziert in Gebiete mit ähnlichen Maisanbauanteilen auf Basis der standörtlichen Eignung für den Maisanbau, den Landbaugebieten. Für das Bundesland Bayern sind die Maisanbaudaten auf Gemeindeebene verfügbar. Diese Daten wurden zu Maisanbauklassen aggregiert, die analog zu den Landbaugebieten in [1], Gebiete mit ähnlichen Maisanbauanteilen repräsentieren. Diese konnten in dieser Form als Eingangsdaten für den Simulationsalgorithmus verwendet werden. Damit ist es möglich den Algorithmus (Abbildung 1a-b) bundesweit einzusetzen. Es wurden die Anbauflächen für Silomais und für Körnermais aus dem Jahr der letzten Agrarstrukturerhebung 2007 herangezogen (Tabelle 1) [6-7]. Für ökologisch angebauten Mais liegen keine Statistiken vor, daher wurde der Anbauanteil an der Gesamtanbaufläche des Mais dem Anteil des ökologischen Landbaus [8] an der landwirtschaftlichen Fläche gleichgesetzt, d.h. vereinfachend wurde angenommen, dass der Anbauanteil des ökologischen Landbaus jede Fruchtart, darunter Mais, in gleichem Maße betrifft. Diese statistischen Anbaudaten des Mais wurden mit der Karte der Verwaltungsgrenzen der BRD [9] verknüpft, um sie für die GIS-Analyse verwenden zu können. Für die Geometrie der Ackerflächen wurde im Hinblick auf die angestrebte bundesweite Übertragbarkeit der Methode auf die Ackerflächen aus dem Datenbestand des Amtlichen TopographischKartographischen Informationssystemes (ATKIS) zurückgegriffen [10] im Gegensatz zu den von [1] verwendeten Feldblöcken aus InVeKos. Die Datenvorbereitung dieser Ackerflächen erfolgte durch Verschnitt mit den Verwaltungsgebieten Bundesland, Landkreise, Gemeinden. Die Geodaten der Schutzgebiete waren bundesweit verfügbar und als Datenvorbereitung wurden die NSG und FFH-Gebiete zusammengeführt und

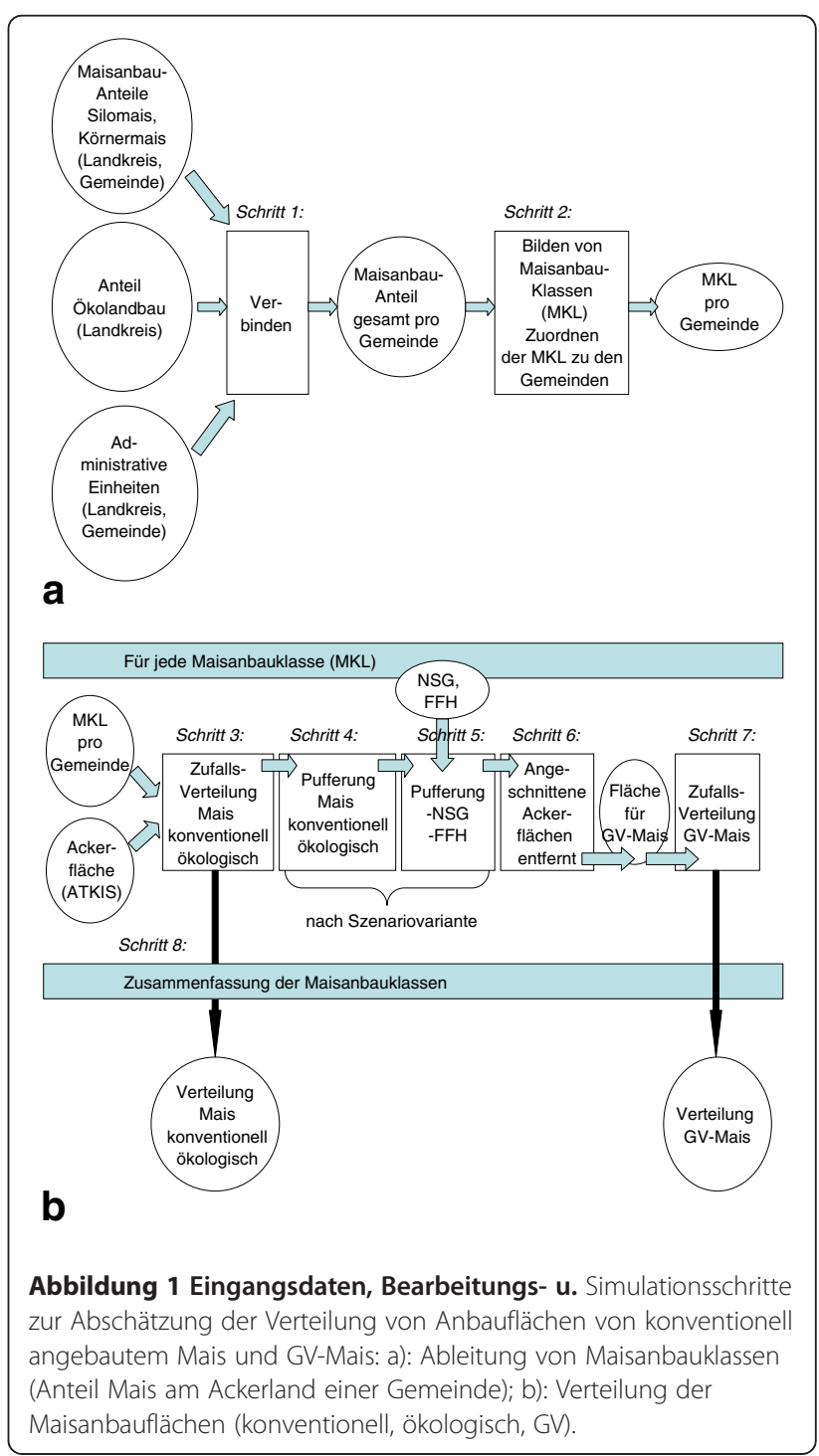


damit eine gemeinsame Pufferung und gemeinde- und landkreisübergreifende Einbeziehung in den GISAlgorithmus ermöglicht.

\section{Gebietsauswahl}

Zur Anwendung und Präsentation der bundesweit einsetzbaren Methode wurde hier exemplarisch das Bundesland Bayern ausgewählt. Es weist in den agrar- und landschaftsstrukturellen Merkmalen eine Vielfalt auf, die in der bundesweiten Übertragung der Methode zu berücksichtigen war, um alle auftretenden räumlichen Situationen in denen Koexistenz gewährleistet werden muss, abbilden und deren Bedeutung diskutieren zu können. Für das Bundesland Bayern galt a) die Maisanbaudaten lagen auf Gemeindeebene vor b) die Maisanbauanteile der Landkreise wiesen eine große Spannbreite auf, von eher niedrigen Maisanbauanteilen im nördlichen, über hauptsächlich mittlere Anteile im mittleren bis zu hohen Anteilen im südlichen Landesteil und c) die mittlere Größe des Ackerlandes lag verglichen mit anderen Bundesländern im mittleren Bereich. Diese Angabe bezieht sich, bedingt durch die Eingangsdaten zur Geometrie der Ackerflächen (Tabelle 1), auf die Ackerflächen aus ATKIS. Einige agrar- und landschaftsstrukturelle Merkmale dieses Bundeslandes, die auch für die Interpretation der Ergebnisse und ihre Einordnung in bisherige Untersuchungen zur Koexistenz wichtig sind [1], zeigt Tabelle 2.

\section{Verschiedene Skalen der Simulation}

Für Bayern wurde die Verteilung von Maisanbauflächen (konventionell und ökologisch angebauter Nicht-GV-Mais, GV-Mais) auf drei verschiedenen Skalen durchgeführt und miteinander verglichen, wobei Vergleiche zur Verteilung: a) auf der Ebene des ganzen Landes gegenüber der auf der Ebene von Landkreisen, anhand dreier ausgewählter
Landkreise und b) auf der Ebene des Landkreises, anhand eines ausgewählten Landkreises gegenüber der auf der Ebene von Gemeinden, anhand zweier Gemeinden dieses Landkreises durchgeführt wurden. Szenarienberechnungen auf unterschiedlichen räumlichen Ebenen bedeuten, dass das für den GV-Anbau zur Verfügung stehende Flächenangebot verschieden ist, in dem einen Fall betrifft es das ganze Bundesland und im anderen Fall steht nur die Fläche des Landkreises zur Verfügung. Die Auswahl der Landkreise erfolgte für a) danach, dass sie zum einen in ihren strukturellen Merkmalen vom Durchschnitt des Bundeslandes abwichen und zum anderen dass die Landkreise möglichst einen unterschiedlichen Maisanbauanteil (Landkreis A, B vs. C) und bei gleichem Maisanbauanteil einen unterschiedlichen Anteil an Schutzgebieten aufwiesen (Landkreis A vs. B), bei nahezu gleicher mittlerer Größe der Ackerflächen. Der Landkreis Kitzingen und die beiden Gemeinden des Landkreises wurden zufällig ausgewählt, um einen Vergleich auch dieser beiden Ebenen zu ermöglichen. Die strukturellen Merkmale der betrachteten Landkreise sind ebenfalls in Tabelle 2 aufgeführt.

\section{Simulation der Maisanbauflächen}

Aus Daten der Anbaustatistiken, kombiniert mit den Geodaten der Ackerflächen wurde die Verteilung der Maisflächen in einem Gebiet folgendermaßen simuliert: Von der Gesamtmaisanbaufläche wurde zunächst die Fläche des Nicht-GV-Mais (konventionell und ökologisch angebauter Mais) auf die Ackerfläche zufallsverteilt, anschließend wurden je nach Szenariovariante die Restriktionen für den GV-Maisanbau eingebunden, d.h. bisher verteilte Nicht-GV-Maisflächen wurden gepuffert und Schutzgebiete mit ihrer Pufferung eingebunden und dann wurden alle Ackerflächen innerhalb der Puffer um

Tabelle 2 Charakterisierung des Bundeslandes Bayern und der untersuchten bayerischen Landkreise: A - Altötting, B Rottal-Inn, C - Bad-Kissingen, D - Kitzingen; Angaben bezogen auf 2007 (Anzahl Gemeinden, Maisanbauklassen, Maisanteil), 2006/08 (Schutzgebiete), 2008 (Ackerfläche); Anteil der Schutzgebiete einschließlich 1000 m Puffer um FFH-Gebiete und NSG

\begin{tabular}{|c|c|c|c|c|c|c|c|c|}
\hline Gebiet & $\begin{array}{c}\text { Anzahl } \\
\text { Gemeinden }\end{array}$ & $\begin{array}{c}\text { Anzahl } \\
\text { Maisanbau- } \\
\text { klassen }\end{array}$ & $\begin{array}{c}\text { Maisanteilan } \\
\text { Ackerfläche }\end{array}$ & $\begin{array}{c}\text { Anteil } \\
\text { Ackerland an } \\
\text { Gebiets-fläche }\end{array}$ & $\begin{array}{c}\text { Ackerland } \\
\text { mittlere Größe }\end{array}$ & $\begin{array}{c}\text { Anteil } \\
\text { Schutz-gebiete } \\
\text { an Gebiets-fläche }\end{array}$ & $\begin{array}{l}\text { Anteil Ackerland } \\
\text { anGebiets-fläche }\end{array}$ & $\begin{array}{l}\text { Anteil Ackerland } \\
\text { e anGebiets-fläche }\end{array}$ \\
\hline & & & & & & & $\begin{array}{c}\text { Ackerland } \\
\text { außerhalb } \\
\text { derSchutz-gebiete }\end{array}$ & $\begin{array}{c}\text { Ackerland } \\
\text { innerhalbderSchutz- } \\
\text { gebiete }\end{array}$ \\
\hline & & & [\%] & {$[\%]$} & [ha] & [\%] & {$[\%]$} & {$[\%]$} \\
\hline Bayern & 1768 & 17 & 20 & 33 & 13 & 37 & 21 & 12 \\
\hline Landkreis A & 24 & 7 & 41 & 44 & 12 & 24 & 34 & 10 \\
\hline Landkreis B & 31 & 5 & 42 & 48 & 14 & 6 & 44 & 3 \\
\hline Landkreis C & 24 & 4 & 5 & 31 & 14 & 49 & 18 & 14 \\
\hline Landkreis D & 29 & 5 & 10 & 55 & 19 & 35 & 36 & 19 \\
\hline
\end{tabular}

${ }^{1}$ Gemeinden mit Ackerfläche $>0$ ha.

${ }^{2}$ basierend auf der Objektart 4101 (Ackerfläche) aus ATKIS. 
die Nicht-GV-Maisflächen und innerhalb der gepufferten Schutzgebiete für die Verteilung des GV-Mais ausgeschlossen und diese erfolgt als letzter Schritt auf den verbliebenen Ackerflächen erneut als Zufallsverteilung. Die Verteilung erfolgte auf den Ackerflächen aus ATKIS (Tabelle 1) und damit wird für die hier durchgeführten Simulationen der Szenarien unterstellt, dass auf diesen Ackerflächen jeweils eine Fruchtart, in diesem Fall Mais, angebaut wird. Aus der Abbildung 1 geht hervor, welche Daten verwendet wurden und in welcher Reihenfolge die Bearbeitungs- und Simulationsschritte erfolgten. Die Erweiterung der Methode zur Simulation der Maisanbauflächen gegenüber [1] betraf die Schritte 1 (Bestimmung der Maisanbauanteile der Gemeinden) und 2 (Zuordnung der Maisanbauanteile der Gemeinden zu Maisanbauklassen).

\section{Schritt 1: Maisanbauanteile der Gemeinden bestimmen}

Die Maisanbauanteile (Silo- und Körnermais) sowie der Anteil des Ökolandbau, die in einer Datenbank vorlagen, wurden über eine Schnittstelle den im GIS vorliegenden Kreisen und Gemeinden zugeordnet. Silomais und Körnermais wurden zum Maisanbauanteil jeder einzelnen Gemeinde zusammengefasst. Angaben über den Anteil an ökologisch angebautem Mais liegen nur auf der Landkreisebene vor und wurden für alle Gemeinden eines Kreises als gleich angenommen. Wenn für eine Gemeinde keine zahlenmäßigen bzw. nur zahlenmäßige Angaben zu Silomais oder Körnermais verfügbar waren, aber eindeutig aus den beiden amtlichen statistischen Kategorien ,Zahlenwert unbekannt' oder ,geheim zu halten $^{\prime 6}$, hervorging, das dort Mais angebaut wurde, wurde aus den vorhandenen Daten (Maisanbau in den anderen Gemeinden bzw. des Landkreises) der für die betreffenden Gemeinden nicht angegebene Maisanbauanteil ermittelt und nach dem vorhandenen Ackerland auf diese Gemeinden verteilt. In 36\% der Gemeinden wurde so entweder der Anbauanteil von Körnermais oder von Silomais ergänzt und in 9\% der Gemeinden gemeinsam der Anbauanteil von Körnermais und Silomais.

\section{Schritt 2: Zuordnung zu Anbauklassen}

In die Szenarioberechnungen wurden die Maisanbauanteile der einzelnen Gemeinden als Mittelwerte von 20 Maisanbauklassen eingegeben, da diese jeweils Gebiete ähnlicher Anbauverhältnisse für den Mais repräsentieren, entsprechend den ,Landbaugebieten', die von Höltl und Wurbs (2008) [1] zur Eingabe von den Maisanbauanteilen, die auf Landkreisebene vorlagen, verwendet wurde. Die Zuweisung der Gemeinden zu Gebieten ähnlicher Maisanbauklassen war Voraussetzung für die Durchführung der nachfolgenden Simulationsschritte. Die, Maisanbauklassen' wurden in 5\%-Schritten gebildet je nach Anteil des Maisanbaus (0 bis 100) an der Ackerfläche einer Gemeinde.

\section{Schritt 3: Räumliche Verteilung der Nicht-GV- Maisanbauflächen}

Aus den Maisanbauklassen auf Gemeindeebene erfolgte auf den (ATKIS-) Ackerflächen eine Zufallsverteilung der Nicht-GV-Maisanbauflächen. Sie entsprechen vom Umfang der Gesamtfläche des Maisanbaus abzüglich der Fläche für den GV-Mais (diese wird im Schritt 7 verteilt). In Schritt 3 wird die Nicht-GV-Maisfläche für die konventionelle und die ökologische Landwirtschaft zufällig verteilt. Die Verteilung der Flächen konventionellen und ökologischen Maisanbaus ist das erste Ergebnis der Simulation.

\section{Schritt 4: Puffer um die Anbauflächen}

Um die (Nicht-GV-)Maisanbauflächen wurde, entsprechend den aktuell gültigen Abstandsregeln, ein Puffer gelegt, $150 \mathrm{~m}$ für konventionell und $300 \mathrm{~m}$ für ökologisch angebauten Mais. Die Anbauflächen wurden je nach betrachteter Simulationsebene (Bundesland, Landkreis oder Gemeinde) gepuffert, nicht jedoch gebietsübergreifend. Damit wurde jeweils das im jeweiligen Szenario definierte Anbauziel und die durch die Abstandsregeln innerhalb des betrachteten Simulationsgebietes auftretenden Restriktionen realisiert.

\section{Schritt 5: Puffer um die Schutzgebiete}

In Simulationen mit Berücksichtigung der Schutzgebiete wurde um die NSG und FFH-Gebiete, entsprechend den z.T. in den Bundesländern gültigen Prüfradien (s.o.), jeweils ein Puffer von 1000 m gelegt. Die Schutzgebiete wurden gemeinde- und landkreisübergreifend, d.h. für das gesamte Bundesland einbezogen und gepuffert, nicht jedoch das Bundesland übergreifend.

\section{Schritt 6: Identifizierung potenzieller GV-Maisflächen}

Die nun mit Nicht-GV-Mais belegten Ackerflächen, die Ackerflächen in den Schutzgebieten (FFH-Gebiete, NSG), die Ackerflächen innerhalb der Pufferzonen, und auch alle Ackerflächen, die von dem Puffer geschnitten werden, stehen für den Anbau von GV-Mais nicht zur Verfügung. Es ergeben sich also hier, als ein Zwischenergebnis, diejenigen Ackerflächen, die genügend Abstand zu NichtGV Mais bzw. Schutzgebieten haben und daher aus dieser Sicht für GV-Mais zur Verfügung stehen.

\section{Schritt 7: Verteilung der GV-Maisflächen}

Auf diesen verbliebenen Ackerflächen erfolgt die Zufallsverteilung von GV-Maisanbauflächen je nach Szenariovariante und damit nach Anteil des GV-Mais an der Gesamtanbaufläche des Mais. Diese modellhafte 
Verteilung des GV-Mais ist ein weiteres Ergebnis der Simulation.

\section{Schritt 8: Zusammenfassung der Simulationen}

Die Schritte 3 bis 7 wurden für jede Maisanbauklasse gesondert durchgeführt, so dass in einem letzten Schritt die Ergebnisse zusammengefasst werden als GIS Karten mit ausgewiesenen Maisanbauflächen. Diese können als Ausgangsbasis zur Identifizierung von Koexistenzproblemen und zur Modellierung der Genflüsse auf Landschaftsebene genutzt werden.

\section{Szenarien}

Als Szenarien werden sowohl unterschiedliche Anbauanteile von GV-Mais (10 und 70\% der Maisfläche) als auch unterschiedliche Berücksichtigung der Schutzgebiete (ohne Berücksichtigung und mit Berücksichtigung der Schutzgebiete einschließlich eines $1000 \mathrm{~m}$ Puffer) berechnet. Der Maisanbauanteil in den einzelnen Gemeinden entspricht jeweils der amtlichen Statistik unter der oben beschriebenen Aggregierung zu Maisanbauklassen. Die Szenarien waren:

- Szenario I: GV-Mais 10\% Anteil, ohne Berücksichtigung der Schutzgebiete

- Szenario II: GV-Mais 70\% Anteil, ohne Berücksichtigung der Schutzgebiete

- Szenario III: GV-Mais 10\% Anteil, mit Berücksichtigung der Schutzgebiete

- Szenario IV: GV-Mais 70\% Anteil, mit Berücksichtigung der Schutzgebiete

Die vier Szenarien wurden sowohl für das gesamte Bundesland Bayern als auch für einzelne Landkreise berechnet. Zum Vergleich der Simulationen des Maisanbaus der Landkreis- mit der Gemeindeebene wurden nur die Szenarien ohne Berücksichtigung der Schutzgebiete gerechnet. Für jedes Szenario wurden fünf Wiederholungen durchgeführt.

\section{Ergebnisse}

Die räumliche Verteilung der Maisanbauflächen einer Simulation des Szenarios III (10\% GV-Maisanteil, mit Berücksichtigung der Schutzgebiete) ist für das Bundesland Bayern (Abbildung 2), den Landkreis Altötting (Abbildung 3) und die Gemeinde Prichsenstadt (Abbildung 4) exemplarisch dargestellt.

Neben der räumlichen Verteilung der verschiedenen Maisanbauflächen zeigen die Abbildungen 2 bis 4 auch die potenziell für den Anbau von GV-Mais zur Verfügung stehenden Ackerflächen. Sie liegen demnach außerhalb der Puffer um die Flächen mit Nicht-GV-Mais und um die Schutzgebiete (FFH-Gebiete, NSG). Wird die Fläche des simulierten angebauten GV-Mais zu der potenziell für den Anbau von GV-Mais zur Verfügung stehenden Fläche ins Verhältnis gesetzt, ermöglicht das

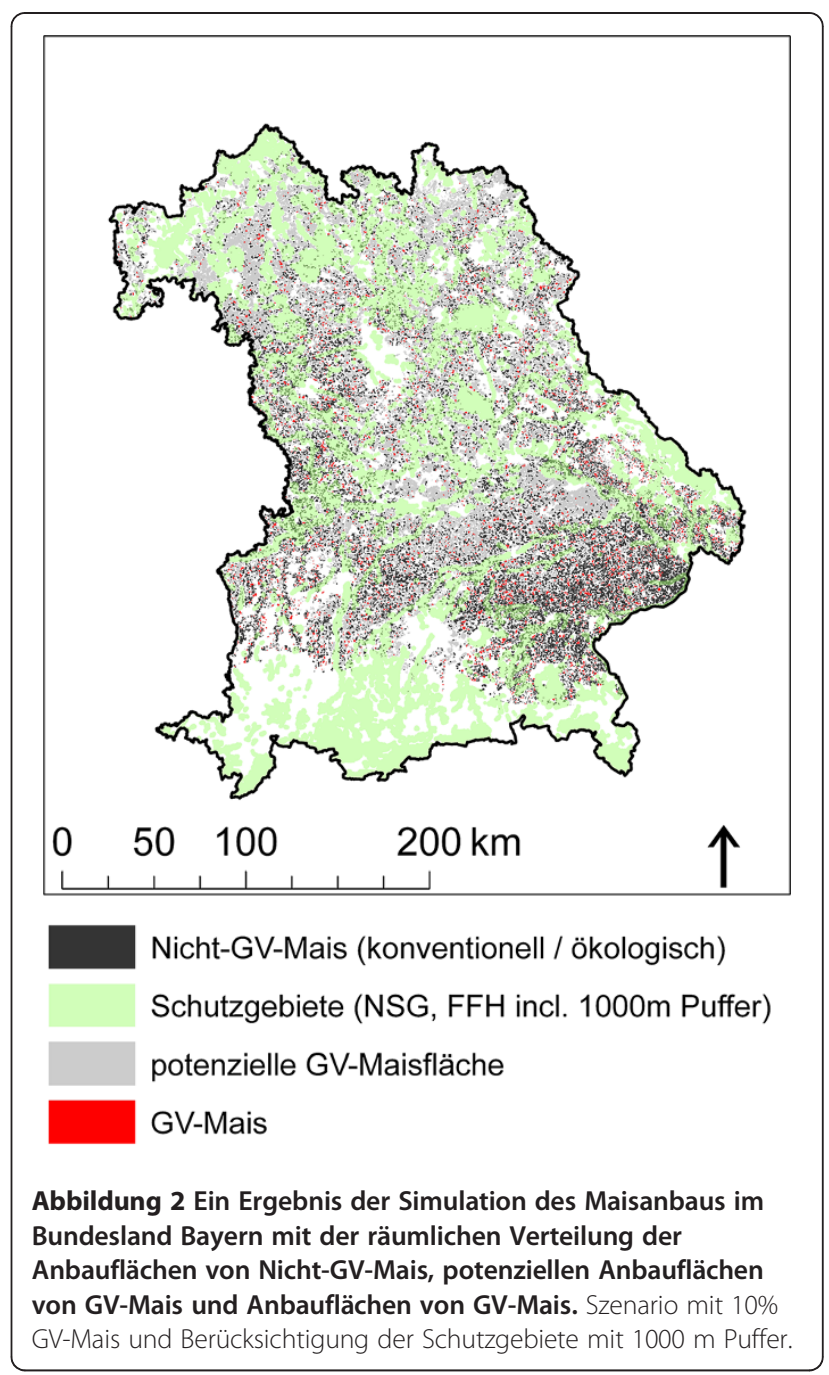

einen Vergleich für die verschiedenen Szenarien, und erlaubt auch Vergleiche sowohl zwischen dem Bundesland Bayern und den drei Landkreisen und den Landkreisen untereinander (Abbildung 5) als auch zwischen dem Landkreis und den beiden zugehörigen Gemeinden (Abbildung 6).

Die Abbildung 5 stellt als Zusammenfassung aller Szenarien dar, wie viel von der zur Verfügung stehenden Fläche tatsächlich für den Anbau von GV-Mais in Anspruch genommen wurde. Die potenziell für den Anbau von GV-Mais zur Verfügung stehende Ackerfläche wird in unterschiedlichem Maße in den Szenarien genutzt, wobei sich die Landkreise deutlich vom Bundesland Bayern und voneinander unterscheiden.

Wie zu erwarten, schöpfen die Szenarien mit einem Anteil von 70\% GV-Mais (sz II, sz IV) diese Fläche stärker aus als die Szenarien mit 10\% GV-Mais (sz I, sz III). Die Berücksichtigung der Schutzgebietsflächen (FFHGebiete, NSG) durch einen 1000 m breiten Puffer um 


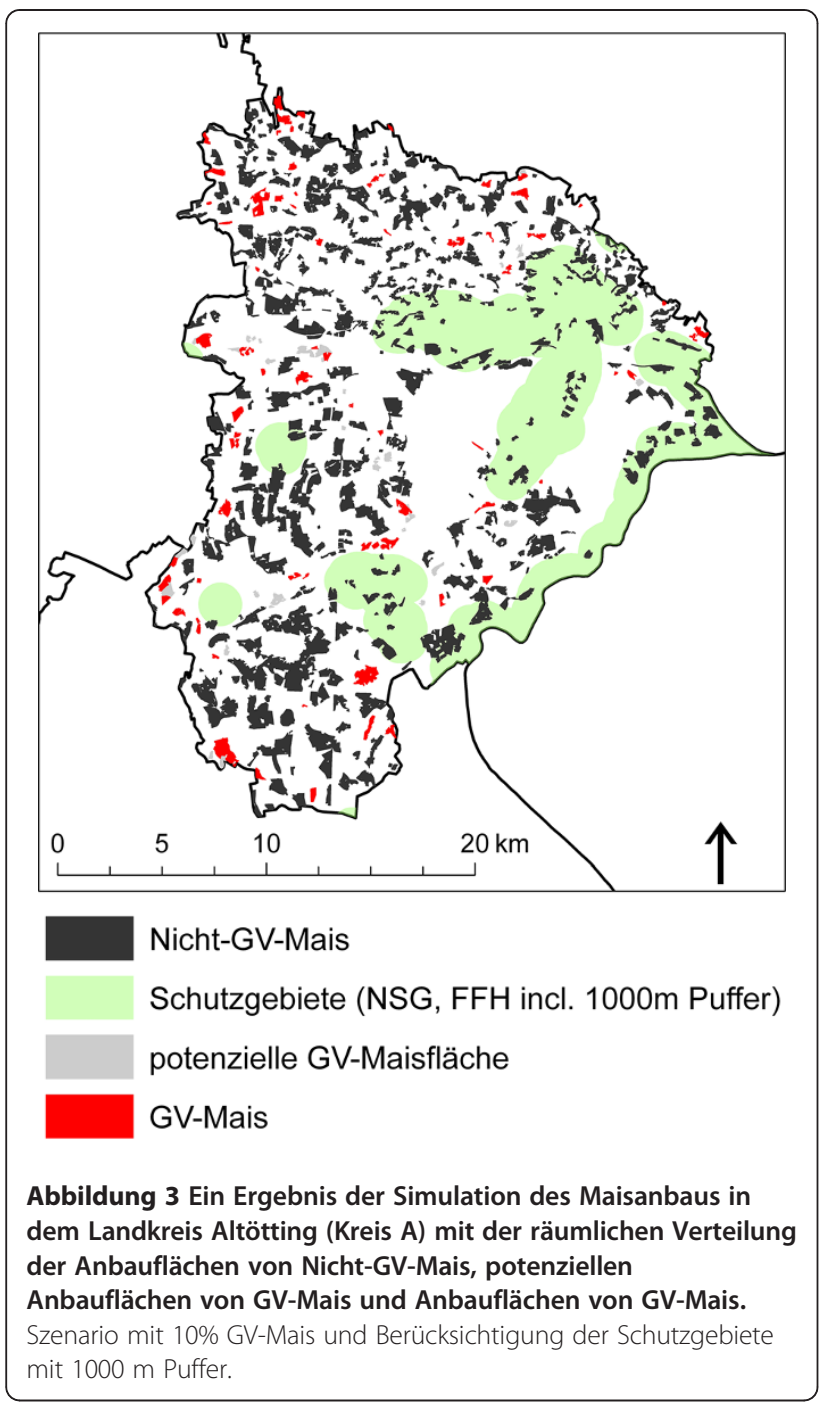

diese Gebiete führt insbesondere beim Landkreis A zu einer Verminderung der für den Anbau von GV-Mais zur Verfügung stehenden Fläche, sowohl bei 10\% GVMais (sz III) als auch bei 70\% GV-Mais (sz IV). Diese Verminderung tritt beim Kreis B nicht auf. Der Kreis A hat mit 24\% der Fläche einen hohen Schutzgebietsanteil gegenüber dem Kreis B (6\%), in dem sich auch ein größerer Anteil der Ackerflächen befindet, bei ansonst fast gleichem Maisanbauanteil. Im Kreis C tritt die Verminderung der potenziellen GV-Mais-Fläche durch die Schutzgebiete nur deutlich bei der Szenariovariante mit 70\% GV-Mais auf (sz IV), da hier der Maisanbauanteil sehr gering ist (5\%).

Unterschiede zwischen den untersuchten Gebieten in der Inanspruchnahme der potenziell für den GV-Mais zur Verfügung stehenden Fläche (Abbildung 5) spiegeln die Unterschiede zwischen den strukturellen Merkmalen der untersuchten Gebiete wieder (Tabelle 2). Während in den Simulationen für das Gesamtgebiet Bayerns die

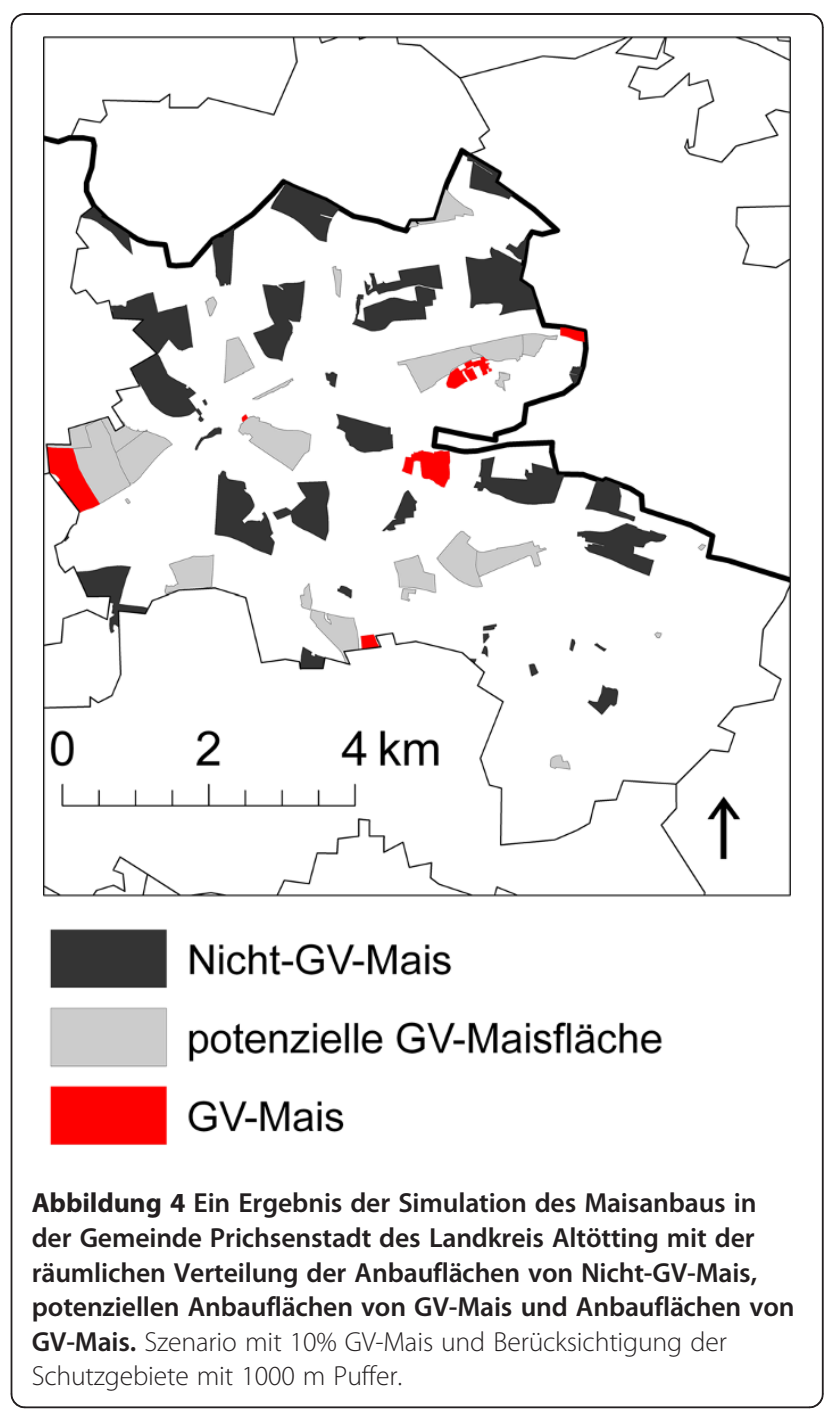

potenzielle Anbaufläche für GV-Mais in allen Szenarien und Wiederholungen nur $\mathrm{zu} 5 \%$ bis 30\% ausgeschöpft wurde, wichen die Landkreise nach oben (Landkreise A und B) und unten (Landkreis C) davon ab (Tabelle 3). Der Grad der Ausschöpfung der potenziell zur Verfügung stehenden Ackerfläche für GV-Mais kann für die hier gewählten Beispielsregionen plausibel mit dem i) Maisanbauanteil: hohe Anteile in den Landkreisen A und $\mathrm{B}$, niedriger Anteil im Landkreis $\mathrm{C}$ korrespondieren mit starker bzw. niedriger Ausschöpfung der potenziell für GV-Mais verfügbaren Ackerfläche durch verteilten GVMais, ii) dem Verhältnis zwischen Ackerlandsanteil und Maisanbauanteil: enges Verhältnis in den Landkreisen A und B, weites Verhältnis im Landkreis C korrespondieren ebenfalls mit starker bzw. niedriger Ausschöpfung der potenziell für GV-Mais verfügbaren Ackerfläche durch verteilten GV-Mais und dem iii) Schutzgebietsanteil: hoher Schutzgebietsanteil Landkreis A und C bewirkt eine erhöhte Inanspruchnahme der potenziell für GV- 

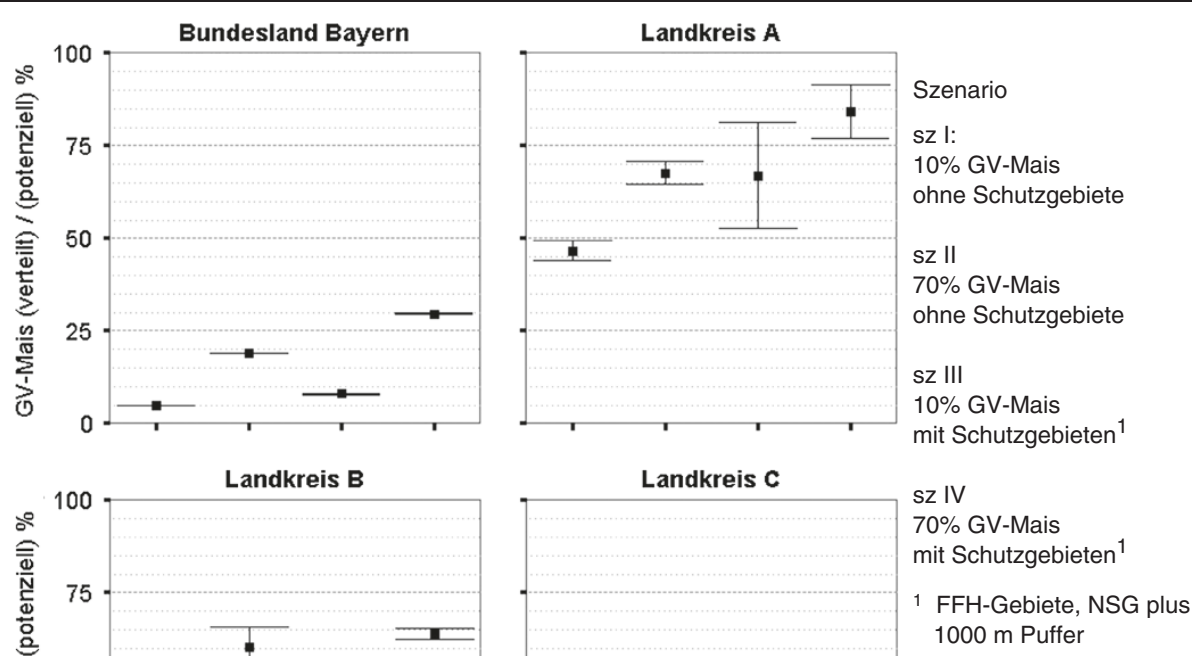

Sz IV

70\% GV-Mais

mit Schutzgebieten ${ }^{1}$

1 FFH-Gebiete, NSG plus $1000 \mathrm{~m}$ Puffer
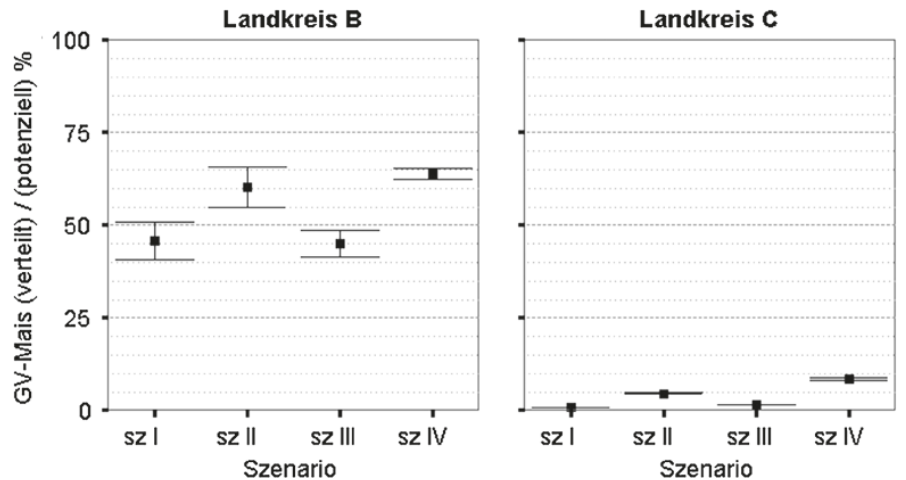

Abbildung 5 Der Anteil des verteilten GV-Mais an der potenziell für den Anbau von GV-Mais zur Verfügung stehenden Ackerfläche $(=100 \%)$ im Bundesland Bayern und drei bayrischen Landkreisen: Altötting (Kreis A), Rottal-Inn (Kreis B), Bad-Kissingen (Kreis C). Die Fehlerbalken entsprechen der einfachen mittleren Standardabweichung von 5 Szenariowiederholungen.

Mais vorhandenen Ackerfläche durch verteilten GVMais in den Szenarien III, IV, insbesondere bei gleichzeitig hohen Maisanbauanteilen wie im Landkreis A (Tabelle 3, Abbildung 5).

In der Abbildung 6 werden die Ergebnisse der Simulation zur Verteilung der Maisanbauflächen in einem bayrischen Landkreis, Kitzingen, und in zwei Gemeinden des Landkreises gegenübergestellt. Für alle drei Gebiete wurde die für den Anbau von GV-Mais zur Verfügung stehende Fläche durch den in der Simulation tatsächlich verteilten GV-Mais in dem Szenario mit dem höheren Anteil an GV-Mais von 70\% (sz II) in größerem Umfang genutzt. Der Landkreis Kitzingen hat mit 10\% im Vergleich mit den drei anderen betrachteten Landkreisen einen eher niedrigen Maisanbauanteil ähnlich dem des Landkreis C mit 5\% (Table 2) und der Anteil der vom simulierten Anbau des GV-Mais beanspruchten Fläche ist mit $2 \%$ bis $10 \%$ ebenfalls etwas höher als im Landkreis C. Die dargestellten zwei Gemeinden des Landkreises zeigen davon abweichende Simulationsergebnisse, indem in der Gemeinde Mainstockheim (Maisanbauklasse 0 bis 5\%) weniger, d.h.

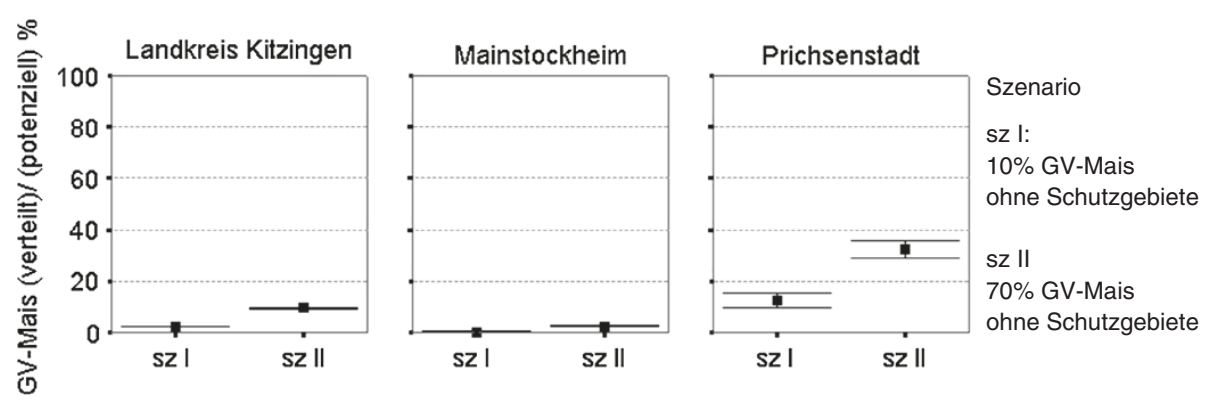

Abbildung 6 Der Anteil des verteilten GV-Mais an der potenziell für den Anbau von GV-Mais zur Verfügung stehenden Ackerfläche $(=100 \%)$ im Landkreis Kitzingen und zwei Gemeinden des Landkreises: Mainstockheim und Prichsenstadt. Die Fehlerbalken entsprechen der einfachen mittleren Standardabweichung von 5 Szenariowiederholungen. 
Tabelle 3 Ausschöpfung der potenziell für GV-Mais verfügbaren Ackerfläche durch in der Szenarienberechnung verteilten GV-Mais in Beziehung zu den agrar- und landschaftsstrukturellen Merkmalen für das Bundesland Bayern und die Landkreise A - Altötting, B - Rottal-Inn, C - Bad-Kissingen

\begin{tabular}{ccccc}
\hline Gebiet & $\begin{array}{c}\text { Ausschöpfung potenziell für GV- } \\
\text { Mais verfügbarer Ackerfläche } \\
\text { durch verteilten GV-Mais }\end{array}$ & $\begin{array}{c}\text { Maisanbauanteil } \\
\text { Mckerlandsanteil/ }\end{array}$ & $\begin{array}{c}\text { Schutzgebietsanteil } \\
\text { Maisanbauanteil }\end{array}$ \\
\hline Bundesland Bayern & {$[\%]$} & {$[\%]$} & {$[-]$} & 2 \\
Landkreis A & 5 bis 30 & 20 & 2 & 37 \\
Landkreis B & 44 bis 92 & 41 & 1 & 24 \\
Landkreis C & 38 bis 66 & 42 & 6 & 6 \\
\hline
\end{tabular}

$0 \%$ bis $2 \%$, und in der Gemeinde Prichsenstadt (Maisanbauklasse 20 bis 25\%) mehr, d.h. 12\% bis 33\% der potenziell für den Anbau von GV-Mais zur Verfügung stehenden Fläche durch die simulierte Verteilung des GV-Mais ausgeschöpft wurde.

Während in den Abbildungen 5 und 6 die Simulationsergebnisse summiert für alle Maisanbauklassen wiedergegeben werden, können die Ergebnisse der Simulation auch im Detail für einzelne
Maisanbauklassen betrachtet werden. Als Beispiel zeigt die Abbildung 7 den Anteil des verteilten GV-Mais an der potenziell für den Anbau von GV-Mais zur Verfügung stehenden Ackerfläche der einzelnen Maisanbauklassen, und zwar für das ganze Bundesland Bayern und wiederum für die Landkreise A, B und C für jeweils eine Wiederholung der Simulation des Szenarios mit 10\% GV-Mais und mit Berücksichtigung der Schutzgebiete (1000 m Puffer).
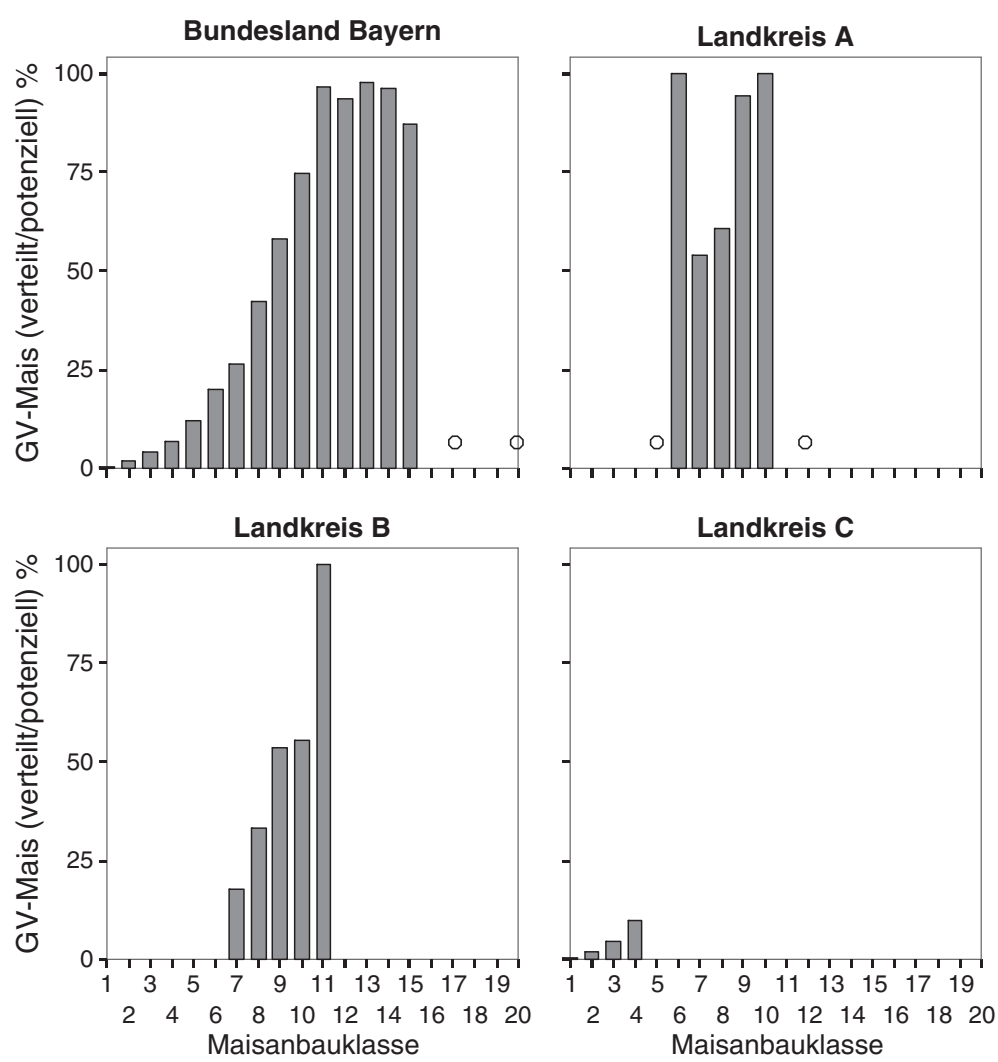

Abbildung 7 Der Anteil des verteilten GV-Mais an der potenziell für den Anbau von GV-Mais zur Verfügung stehenden Ackerfläche $(=100 \%)$ der einzelnen Maisanbauklassen für das Bundesland Bayern und die drei bayrischen Landkreise: Altötting (Kreis A), Rottal-Inn (Kreis B), Bad-Kissingen (Kreis C). Die Simulation erfolgte nach dem Szenario III mit 10\% GV-Mais und mit Berücksichtigung der Schutzgebiete. Hier ist jeweils die Szenariowiederholung 1 wiedergegeben. Die Kreise markieren Maisanbauklassen in denen während der Simulation kein GVMais verteilt wurde. 
Die Landkreise bestehen aus 24 (Landkreis A), 31 (Landkreis B) und 24 (Landkreis C) Gemeinden, in denen die Anbauanteile von Mais zwischen 24,7 und 59,8\% (Landkreis A), zwischen 30,7 und 54,4\% (Landkreis B) und zwischen 0 und $16,1 \%$ (Landkreis C) lagen und die dementsprechend durch sieben Maisanbauklassen: 5 - 10, 12 (Landkreis A), fünf Maisanbauklassen: 7 - 11 (Landkreis B) und vier Maisanbauklassen: 1 - 4 (Landkreis C) repräsentiert waren, während es im Land Bayern bei 1768 Gemeinden, mit Anbauanteilen von Mais zwischen 0 und 100\%, 17 Maisanbauklassen (1 - 15, 17 und 20) gab (Abbildung 7).

In der Simulation für das ganze Bundesland Bayern war nach der Verteilung der Nicht-GV-Maisflächen (nach Schritt 6 des GIS-Algorithmus) in den Gemeinden mit den Maisanbauklassen 17 und 20 keine Ackerfläche mehr für den GV-Maisanbau verfügbar. Dies trifft auch für einzelne Landkreise zu, z.B. den Kreis A in zwei Maisanbauklassen $(5,12)$.

Die potenziell für den Anbau von GV-Mais zur Verfügung stehende Ackerfläche wurde in der Simulation für das gesamte Land in den Maisanbauklassen 11 bis 15, also ab einem Maianbauanteil von 52,5\% jeweils zu nahezu 95\% oder mehr ausgeschöpft. In diesen Klassen war die potenziell zur Verfügung stehende Fläche kleiner als die Fläche die für eine Verteilung von 10\% GV-Mais benötigt worden wäre (Klasse 11, 13, 14) oder die verbliebenen einzelnen Restflächen waren zu groß, um in die Flächen für die Verteilung von GV-Mais noch einbezogen werden zu können (in Klasse 12 waren 17 ha und in Klasse 15 waren 3 ha übrig). In der Simulation für den Landkreis A wurde die potenziell für den Anbau von GV-Mais zur Verfügung stehende Ackerfläche in drei Maisanbauklassen (Klasse 6, 9, 10) zu mehr als 95\% durch die Verteilung von Anbauflächen für GV-Mais ausgeschöpft. Diese Klassen entsprechen einem Maisanbauanteil von 27,5\%, $42,5 \%$ und $47,5 \%$. Hier war auch entweder die potenziell zur Verfügung stehende Fläche kleiner als die Fläche die für eine Verteilung von 10\% GV-Mais benötigt worden wäre (Klasse 6, 10) oder die verbliebenen einzelnen Restflächen waren $\mathrm{zu}$ groß, um in die Flächen für die Verteilung von GV-Mais noch einbezogen zu werden (in Klasse 9 waren 5 ha übrig). In der Simulation für den Landkreis B wurde die potenziell für den Anbau von GV-Mais zur Verfügung stehende Ackerfläche nur in der Maisanbauklasse $11 \mathrm{zu}$ mehr als 95\% durch die Verteilung von Anbauflächen für GV-Mais ausgeschöpft, was einem Maisanbauanteil von 52,5\% entspricht. In dem Landkreis $\mathrm{C}$ wurde hingegen in keiner Maisanbauklasse die potenziell für den Anbau von GV-Mais zur Verfügung stehende Ackerfläche zu mehr als 95\% durch die Verteilung von Anbauflächen für GV-Mais ausgeschöpft, sie waren für alle Maisanbauklassen nicht größer als $10 \%$.

\section{Diskussion}

Die Simulation zur Verteilung von Anbauflächen des mittels konventionell und ökologischer Anbauweise angebauten Mais und des GV-Mais unter definierten Rahmenbedingungen (Anbauanteil des GV-Mais, Beachtung von Mindestabständen zu Nicht-GV-Mais und Schutzgebieten, statistisch erfasster Maisanbauanteil auf Basis der Gemeinden) resultiert in jeweils möglichen räumlichen Verteilungsmustern der Anbauflächen, die unter den Koexistenzbedingungen realisiert werden können (Abbildungen 2 bis 4). Jede Simulation stellt eine Lösungsmöglichkeit für Koexistenz dar. Unter den unterschiedlichen Szenariobedingungen ist eine jeweils charakteristische Verteilung der Anbauflächen zu erwarten, z.B. unter Berücksichtigung der Abstände zu Schutzgebieten bilden sich clusterartige Anbauschwerpunkte des GV-Mais außerhalb von Schutzgebieten, insbesondere bei hohen Anbauanteilen von GV-Mais heraus. Die räumlichen Verteilungsmuster können zur großräumigen (Abbildung 2), regionalen (Abbildung 3) oder lokalen (Abbildung 4) Modellierung von Genflüssen zwischen Anbauflächen von GV-Mais und Nicht-GVMais verwendet werden [5].

Ein weiteres Ergebnis der Simulation ist das Verhältnis der Anbaufläche des GV-Mais, die in der Simulation tatsächlich verteilt werden konnte, zur potenziell für eine Verteilung von GV-Mais zur Verfügung stehenden Fläche, die sich aus der Simulation der Verteilung der Nicht-GV-Maisflächen und unter Beachtung von Mindestabständen zu Nicht-GV-Maisflächen und zu Schutzgebieten ergibt. Wird die potenziell für eine Verteilung von GV-Mais zur Verfügung stehende Fläche stärker durch die tatsächlich verteilte GV-Maisfläche ausgeschöpft bzw. überschritten, so resultiert daraus ein größeres räumliches Konfliktpotenzial.

Die Ergebnisse legen nahe, dass dieses Flächenverhältnis von Faktoren beeinflusst wird, die einerseits in den Szenariovarianten erfasst sind, wie Anbauanteil von GVMais, Berücksichtigung bzw. Nichtberücksichtigung von Abständen zu Schutzgebieten (Abbildungen 5 und 6) und andererseits in den agrar- und landschaftsstrukturellen Eigenschaften der Gebiete begründet sind, wie Maisanbauanteil, Verhältnis zwischen Ackerlandsanteil und Maisanbauanteil, Schutzgebietsanteil (Tabelle 2, Abbildungen 5 und 6). In Gebieten mit hohem Nutzungsdruck auf den Agrarflächen durch hohen Anbauanteil an GV-Mais, hohen $\mathrm{zu}$ berücksichtigenden Schutzgebietsanteilen, hohem Maisanbauanteil ist ein größeres räumliche Konfliktpotenzial bei der Umsetzung der Koexistenz zwischen Nicht-GV-Mais und GV-Mais zu erwarten. Die Faktoren Maisanbauanteil und Anbauanteil GV-Mais haben auch nach Devos et al. (2007) [2] einen Einfluss auf die Umsetzbarkeit der Koexistenz. Unabhängig von den getesteten Szenarien wird ein Einfluss des 
Maisanbauanteils auf diese Koexistenzmöglichkeiten diskutiert. In den Szenarien selbst, wird darüber hinaus der Einfluss des GV-Maisanteils untersucht; auch hier nimmt die Umsetzungsmöglichkeit der Koexistenz bei höherem Anteil ab. Die mittleren Feldgrößen mit 1,2 ha in der Koexistenzstudie von Devos et al. (2007) [2] waren im Vergleich zu denen unserer untersuchten Gebiete (Tabelle 2) geringer, trotzdem weisen beide Untersuchungen hinsichtlich der genannten Einflussfaktoren vergleichbare Tendenzen auf. Devos et al. (2007) [2] schlussfolgern, dass insbesondere ein hoher Maisanbauanteil die räumliche Trennung und damit die Koexistenz von Nicht-GV-Mais und GV-Mais erschwert und empfehlen als effektive Strategie zur räumlichen Abgrenzung die Konzentration von GV-Maisflächen in größeren Gebieten. Jedoch führt letzteres in unseren vorgestellten Szenarien unter Berücksichtigung der Abstände zu Schutzgebieten zur Erhöhung des räumlichen Konfliktpotenzials auf den Flächen außerhalb der gepufferten Schutzgebiete. Der Maisanbauanteil eines Gebietes wird auch von Sanvido et al. (2008) [3] als ein wichtiger Faktor für die Umsetzung der Koexistenz genannt.

Zur Identifizierung von Gebieten, in denen die Umsetzung der Koexistenz unter den gegebenen Rahmenbedingungen tatsächlich Schwierigkeiten bereiten könnte, ist es notwendig, die Ergebnisse der Szenarioberechnung im Detail zu betrachten, d.h. für die Maisanbauklassen jeweils das Verhältnis der tatsächlich verteilten Anbaufläche des GV-Mais zur potenziell für eine Verteilung von GV-Mais zur Verfügung stehenden Fläche (Abbildung 7). Insbesondere können hier auf regionaler und lokaler Ebene diejenigen Gebiete identifiziert werden, in denen die Umsetzung der Koexistenz unter den gewählten Rahmenbedingungen räumlich an ihre Grenzen stößt oder gar nicht mehr möglich ist.

Die Simulationsergebnisse, d.h. das räumliche Konfliktpotenzial stellt sich zwischen den betrachteten Simulationsebenen Bundesland vs. Landkreis und Landkreis vs. Gemeinde unterschiedlich dar. Zur Interpretation sollten deshalb nur Ergebnisse der jeweils gleichen Simulationsebene miteinander verglichen werden. Die großräumige Simulation auf der Ebene des Bundeslandes sollte nur zum Vergleich der Koexistenzmöglichkeiten untereinander verwendet werden. Auf der jeweils unteren räumlichen Ebene können detaillierter Gebiete mit einem möglichen räumlichen Konfliktpotenzial identifiziert werden. Die Simulation auf regionaler Ebene, mit relativ homogenen agrar- und landschaftsstrukturellen Merkmalen und einer realitätsnäheren Begrenzung der für die Verteilung von Anbauflächen zur Verfügung stehenden Ackerfläche, ist daher geeignet, über eine Regionalisierung potenzielle Konfliktgebiete der Koexistenz auch für größere Gebiete zu identifizieren. Auf lokaler Ebene können diese Gebiete weiter eingegrenzt werden und tatsächliche Grenzen der
Koexistenz auf Gemeindeebene gezeigt und mögliche räumliche Lösungen mit den zur Verfügung stehenden Flächen gefunden werden. Die Beschränkung auf ein kleineres Anbaugebiet ist realitätsnäher, da auch die Anbauentscheidungen eines landwirtschaftlichen Betriebes eher auf dieser Ebene getroffen werden.

Die Methode zur Simulation von Maisanbauflächen zur Abschätzung der Möglichkeiten der Koexistenz zwischen Nicht-GV-Mais und GV-Mais unter verschiedenen Koexistenzbedingungen von Höltl und Wurbs (2008) [1] wurde so weiterentwickelt, dass sie bundesweit übertragbar ist und auf unterschiedlichen räumlichen Ebenen (Bundesland, Landkreis, Gemeinde) gerechnet werden kann. Für eine bundesweite Abschätzung des räumlichen Konfliktpotenzials, dass sich aus der Umsetzung der Koexistenz ergeben kann, bietet die Methode zwei Wege a) die Simulation auf der Ebene des Bundeslandes, b) die Simulation auf der Ebene des Landkreises und die anschließende Regionalisierung der Simulationsergebnisse über den Zusammenhang der Simulationsergebnisse zu den agrar- und landschaftsstrukturellen Merkmalen der Landkreise. Auf Grund der oben dargestellten Zusammenhänge ist Variante $b$ vorzuziehen. Der enge Zusammenhang zwischen dem betrachteten Landschaftsausschnitt und der Umsetzungsmöglichkeit der Koexistenz und die kleinräumig wechselnden Einflussfaktoren der Koexistenz, wie z.B. Maisanbauanteil [3] können so besser berücksichtigt werden. Die Methode ist flexibel, d.h. es können veränderte Rahmenbedingungen (z.B. Mindestabstände, Anbauanteile), detailliertere Datengrundlagen (z.B. genauere Geometrien von Anbauflächen auf lokaler Ebene) eingebunden werden und sie kann auf andere GV-relevante Fruchtarten (z.B. Raps, Zuckerrüben) angewandt werden.

Die hier vorgestellte Methode vollzieht mit dem angewendeten GIS-Algorithmus (Abbildung 1b), die Reihenfolge der Anbauentscheidungen nach, wie sie in der Anbaupraxis durchgeführt werden. Das bedeutet: a) zunächst entscheiden die Landwirte über den Anbau von Mais nach konventioneller und ökologischer Anbauweise und b) anschließend müssen diejenigen Landwirte die GV-Mais anbauen wollen, diese Anbaustandorte bei ihrer eigenen Anbauentscheidung mit Hilfe von Mindestabständen ermitteln und berücksichtigen (\$§ 3,4 GenTPflEV 2008 ${ }^{4}$ ). Diese Vorgehensweise unterscheidet sich in dieser Reihenfolge von denjenigen Koexistenzstudien, z.B. Devos et al. (2007) [2], die zunächst die Anbauflächen von GV-Mais verteilen und um diese die Pufferflächen zur Einhaltung der Abstandsregeln legen. Eine Weiterentwicklung der Methode sollte über die Berücksichtigung der Reihenfolge der Anbauentscheidungen hinaus auch die Tatsache berücksichtigen, dass Landwirte diejenigen Flächen die nur teilweise innerhalb 
der Mindestabstandsdistanz zu Nicht-GV-Maisflächen liegen, auch teilen können, um auf den außerhalb liegenden Flächen GV-Mais anbauen zu können. Damit könnte das Konfliktpotenzial vermindert, d.h. die für den Anbau von GV-Mais potenziell zur Verfügung stehende Fläche vergrößert werden.

\section{Schlussfolgerungen}

Die vorgestellte Methode ist geeignet, um die räumliche Verteilung von Anbauflächen von Nicht-GV-Mais und GVMais unter frei wählbaren Rahmenbedingungen wie Anteil des GV-Mais, Berücksichtigung von Mindestabständen um Flächen von Nicht-GV-Mais, Berücksichtigung von Schutzgebieten, auf unterschiedlichen räumlichen Ebenen zu simulieren.

Eine Übertragbarkeit auf andere Fruchtarten, das Berücksichtigen zusätzlicher oder anderer Rahmenbedingungen ist unter Beibehaltung der Grundmethodik möglich. Ebenso denkbar ist, die Simulation auf Gemeindeebene mit Betriebsdaten als Ausgangsdaten für die Ackerflächen und den Anbau durchzuführen, um die Ackerschläge genauer als es mit den ATKIS-Daten möglich ist, einzubeziehen. Ein solcher Datenbestand kann evtl. aus betrieblichen Einzelerhebungen stammen.

Die Simulation auf der Ebene eines Bundeslandes kann nur erste Hinweise darauf geben, in welchen Maisanbauklassen allein die räumliche Konstellation der Ackerflächen und Schutzgebiete für die Umsetzung der Koexistenz begrenzend wirkt. Wird die Simulation auf der Ebene eines Bundeslandes durchgeführt, ist die erreichte Verteilung der Anbauflächen von konventionellem Mais, ökologisch angebautem Mais und GV-Mais jedoch nutzbar für weitere Analysen, wie z.B. die Modellierung des Genflusses zwischen den Maisanbauflächen für ein Bundesland. Auf Basis dieser Modellierung können dann Problemgebiete für die Koexistenz zwischen den Anbauformen ausgewiesen werden, siehe z.B. Reuter et al. (2008) [5].

Die Simulation auf der Ebene eines Landkreises bietet genauere Hinweise in welchen Maisanbauklassen und damit in welchen Gemeinden die Umsetzung der Koexistenz zwischen dem Anbau von konventionellem Mais, ökologischem Mais und GV-Mais durch die räumliche Situation problematisch sein könnte, in Abhängigkeit der Anbauanteile von GV-Mais. Die Simulation auf dieser regionalen Ebene ermöglicht also Aussagen zur Umsetzbarkeit der Koexistenz in den Landkreisen. Da in den Landkreisen die räumliche Ausstattung homogener ist als im ganzen Bundesland, wird dadurch eine Verknüpfung von Aussagen zur Koexistenz mit der Landschaftsstruktur ermöglicht und eine Übertragbarkeit von Aussagen auf ähnlich ausgestattete Landkreise, für die keine Szenarioberechnung durchgeführt wurden. Eine regionale Modellierung des Genflusses ist auf Basis der
Verteilung der Anbauflächen von konventionellem Mais, ökologisch angebautem Mais und GV-Mais innerhalb des Landkreises ebenso möglich.

Mit einer Simulation auf der Ebene einer Gemeinde können lokal die Grenzen der Koexistenzmöglichkeiten für diejenigen Gebiete genauer untersucht werden, für die Hinweise auf ein höheres räumliches Konfliktpotenzial aus der Simulationsrechnung auf der Ebene des Landkreises existieren. Ebenso kann eine Simulation auf Ebene der Gemeinden helfen, räumliche Lösungsmöglichkeiten zur Umsetzung der Koexistenz $\mathrm{zu}$ finden. Eine lokale Modellierung des Genflusses ist auf Basis der Verteilung der Anbauflächen von konventionellem Mais, ökologisch angebautem Mais und GV-Mais könnte darauf aufbauend innerhalb einer Gemeinde weitere Hinweise zur geeigneten Verteilung der Anbauflächen ermöglichen.

\section{Fußnoten}

1 Richtlinie 2001/18/EG des Europäischen Parlaments und des Rates vom 12. März 2001 über die absichtliche Freisetzung genetisch veränderter Organismen in die Umwelt und zur Aufhebung der Richtlinie 90/220/EWG des Rates; http://eur-lex.europa.eu/LexUriServ/LexUriServ. 847do?uri=OJ:L:2001:106:0001:0038:DE:PDF; geändert durch 848Artikel 43 aus Verordnung (EG) Nr. 2003 des 849Europ/2003 des 849Europäischen Parlaments und des Rates vom 22. Sep- 850tember 2003 über genetisch veränderte Lebensmittel und 851Futtermittel; http://eurlex.europa.eu/LexUriServ/site/de/852consleg/2003/R/ 02003R1829-20070112-de.pdf

2 GenTG (Gentechnikgesetz - Gesetz zur Regelung der Gentechnik i.d.F. vom 16. Dezember 1993 (BGBl. I S. 2066), zuletzt geändert am 01. April 2008 (BGBl. I S. 499)

3 GenTPflEV (Verordnung über die gute fachliche Praxis bei der Erzeugung gentechnisch-veränderter Pflanzen - Gentechnik-Pflanzenerzeugungsverordnung i. d.F. vom 7. April 2008 (BGBl. I S. 655)

4 Runderlass des Brandenburger Ministeriums für Ländliche Entwicklung, Umwelt und Verbraucherschutz vom 27. März 2008; http://www.brandenburg.de/cms/ media.php/2318/bt_mais.pdf

${ }^{5}$ BayNatSchG (Gesetz über den Schutz der Natur, die Pflege der Landschaft und die Erholung in der freien Natur (Bayerisches Naturschutzgesetz - BayNatSchG) vom 23. Februar 2011; https://www.verkuendung-bayern. de/gvbl/jahrgang:2011/heftnummer:4/seite:82

${ }^{6}$ Dies ist in einigen Gemeinden aus Gründen des Datenschutzes erforderlich

\section{Abkürzungsliste}

ATKIS: Amtliches Topographisch- Kartographisches Informationssystem;

GIS: Geographisches Informationssystem; GV-Mais: Mit Mitteln der Gentechnik erzeugter Mais; FFH-Gebiet: Schutzgebiet des Netzes Natura 2000 nach der

Fauna-Flora-Habitat-Richtlinie (Richtlinie 92/43/EWG, zuletzt geändert durch 
die Richtlinie 2006/105/EG) zur Erhaltung der natürlichen Lebensräume sowie der wildlebenden Tiere und Pflanzen; InVeKoS: Integriertes Verwaltungs- und Kontrollsystem; NSG: Naturschutzgebiet nach $\S 23$ des

Bundesnaturschutzgesetz.

\section{Interessenkonflikte}

Die Autoren erklären, dass sie keine Interessenskonflikte haben.

\section{Beitrag der Autoren}

AW hat die grundlegende Methode des GIS-Algorithmus entwickelt und an der Weiterentwicklung mitgearbeitet, CB hat die Methode für die bundesweite Übertragung und für die Anwendung auf verschiedenen räumlichen Skalen weiterentwickelt, angewandt und ausgewertet, US war an der Auswertung beteiligt und hat den ersten Entwurf des Manuskriptes erstellt. Alle drei Autoren haben den Text gelesen und das fertige Manuskript erstellt.

\section{Danksagung}

Das vorliegende Manuskript entstand im Rahmen des Forschungsprojektes GeneRisk, das sich mit den ökologischen, juristischen und ökonomischen Analysen der Koexistenz von Landwirtschaft mit und ohne gentechnisch veränderten Pflanzen befasste. Die Autoren danken dem Bundesministerium für Bildung und Forschung (BMBF) für die finanzielle Unterstützung des Forschungsprojektes GeneRisk (FKZ: 07VPS14A-F), sowie dem Bundesministerium für Ernährung, Landwirtschaft und Verbraucherschutz (BMELV) und dem Ministerium für Infrastruktur und Landwirtschaft des Landes Brandenburg (MIL).

Received: 19 August 2011 Accepted: 27 March 2012

Published: 16 May 2012

\section{References}

1. Höltl K, Wurbs A: Simulation of GM maize-cultivation scenarios under different coexistence regulations. In Implications of GM-Crop Cultivation at Large Scales. Edited by Breckling B, Reuter H, Verhoeven R. Theorie in der Ökologie 14 2008. Frankfurt, Peter Lang, pp 43-46

2. Devos Y, Reheul D, Thas O, De Clercq EM, Cougnon M, Cordemanns K: Implementing isolation perimeters around genetically modified maize fields. Agron Sustain Dev 2007, 27:11-30. doi:10.1051/agro:2007005.

3. Sanvido O, Widmer F, Winzeler M, Streit B, Szerencsits E, Bigler F: Definition and feasibility of isolation distances for transgenic maize cultivation. Transgenic Res 17 2008: 317-335. doi: 10.1007/s11248-007-9103-1

4. Devos Y, Demont M, Dillen K, Reheul D, Kaiser M, Sanvido: Coexistence of genetically modified (GM) and non-GM crops in the European Union - a review. Agron Sustain Dev 2009, 29:11-30. doi:10.1051/agro:2008051.

5. Reuter H, Breckling B, Wurbs A, Höltl K: Modelling maize cross-pollination probabilities on the regional level - exemplary simulations for the county Elbe Elster in Brandenburg, Gemany. In: Implications of GM-Crop Cultivation at Large Scales. Edited by Breckling B, Reuter H, Verhoeven R. Theorie in der Ökologie 14 2008. Frankfurt, Peter Lang, pp 54-58

6. Bayrisches Landesamt für Statistik und Datenverarbeitung: Genesis Online Bayern. [https://www.statistikdaten.bayern.de/genesis/online/logon (download 27.07.2009)]

7. Statistische Ämter des Bundes und der Länder2009Statistik lokal, Daten für die Gemeinden, kreisfreien Städte und Kreise DeutschlandsIT. NRWDüsseldorfStatistische Ämter des Bundes und der Länder: Statistik lokal, Daten für die Gemeinden, kreisfreien Städte und Kreise Deutschlands. Düsseldorf: IT.NRW; 2009.

8. Statistische Ämter des Bundes und der Länder: Regionaldatenbank Deutschland, Genesis 2009 [https:/www.regionalstatistik.de/genesis/online/ logon (download 14.07.2009)]

9. Vermessungsverwaltungen der Länder und BKG: Verwaltungsgrenzen BRD 2007, 2007 Frankfurt am Main

10. Vermessungsverwaltungen der Länder und BKG: ATKIS, Basis-DLM, 2008 Frankfurt am Main

11. Bayerisches Landesamt für Umwelt: Schutzgebietsdaten/Ökoflächen aus dem Bayerischen Fachinformationssystem Naturschutz (FIS-Natur) 2009

doi:10.1186/2190-4715-24-17

Cite this article as: Wurbs et al:: Assessment of regional capabilities for agricultural coexistence with genetically modified maize. Environmental Sciences Europe 2012 24:17

\section{Submit your manuscript to a SpringerOpen ${ }^{\mathcal{D}}$ journal and benefit from:}

- Convenient online submission

- Rigorous peer review

- Immediate publication on acceptance

- Open access: articles freely available online

- High visibility within the field

- Retaining the copyright to your article

Submit your next manuscript at $>$ springeropen.com 